\title{
Brightness variations of young Sun-like stars from ground-based and space telescopes
}

\author{
G. Zsidi ${ }^{1}$, Á. Kóspál ${ }^{1,2}$, P. Ábrahám ${ }^{1}$, R. Szabó $^{1}$, B. Cseh ${ }^{1}$, \\ K. Sárneczky ${ }^{1}$, Á. Sódor ${ }^{1}$, R. Szakáts ${ }^{1}$, K. Vida ${ }^{1}$ and J. Vinkó ${ }^{1}$ \\ ${ }^{1}$ Konkoly Observatory, \\ Research Centre for Astronomy and Earth Sciences of the Hungarian Academy of Sciences, \\ Konkoly-Thege Miklós út 15-17, H1121, Budapest, Hungary \\ email: zsidi.gabriella@csfk.mta.hu \\ ${ }^{2}$ Max Planck Institute for Astronomy, \\ Königstuhl 17, D-69117, Heidelberg, Germany
}

\begin{abstract}
Young stellar objects often show photometric variability, which is well examined at optical wavelengths, but more and more infrared data are also available. The wavelength dependence of the variability carries information on the physical cause of the changing brightness. Here, we examine seven T Tauri-type stars known for their large amplitude variability selected from the Campaign 13 field of the Kepler K2 mission. We complemented the K2 light curves by multifilter optical monitoring observations made with the $90 \mathrm{~cm}$ Schmidt telescope of Konkoly Observatory, and by 3.6 and $4.5 \mu \mathrm{m}$ infrared photometry with a 20 hours cadence using the Spitzer Space Telescope. We found that the wavelength dependence of the observed variability is not consistent with changing interstellar extinction. We suggest that the brightness changes are due to variable accretion, causing a variable illumination of the inner disk.
\end{abstract}

Keywords. stars: pre-main-sequence, circumstellar matter

T Tauri stars are young Sun-like stars, which are still being built up by accreting mass from the surrounding circumstellar disk made of gas and dust. They often show photometric variability (e.g. Herbst et al. 1994, Morales-Calderón et al. 2011), which may be due to non-steady accretion from the circumstellar disk onto the star, but other processes, such as rotating cold or hot spots, or eclipses caused by dust clouds moving in and out of the line-of-sight can also change the observed brightness. The observations at different wavelengths trace different regions and processes in the system, and they are all connected through the disk (Sicilia-Aguilar et al. 2016).

We selected seven T Tauri-type stars (Haro 6-10, HBC 393, HL Tau, XZ Tau, UZ Tau E, DR Tau and DQ Tau) from the Campaign 13 field of the Kepler K2 mission, which covered part of the Taurus star forming region. We obtained high precision optical photometry with 1 minute cadence between 2017 March 8 and May 27. In order to extend the wavelength coverage, we complemented the K2 data with 3.6 and $4.5 \mu \mathrm{m}$ infrared observations at a 20 hours cadence using the Spitzer Space Telescope between 2017 May 17 and May 28. We also carried out monitoring observation in $B, V, R_{C}$ and $I_{C}$ bands with the 90 cm Schmidt telescope of Konkoly Observatory (Hungary) between 2017 March 8 and April 10. Here, we present results on two of our targets: DR Tau and HL Tau.

DR Tau is a highly accreting classical T Tauri star. The K2 light curve (Kp magnitudes are plotted in Fig. 1) shows variability on hourly timescale. Stochastic variations with peak-to-peak amplitude of $1.4 \mathrm{mag}$ appear on this light curve. In contrast, HL Tau is a deeply embedded classical T Tauri star. Its K2 light curve (Fig. 2) shows small amplitude variations on a timescale of a few days with peak-to-peak amplitude of 0.3 mag. 


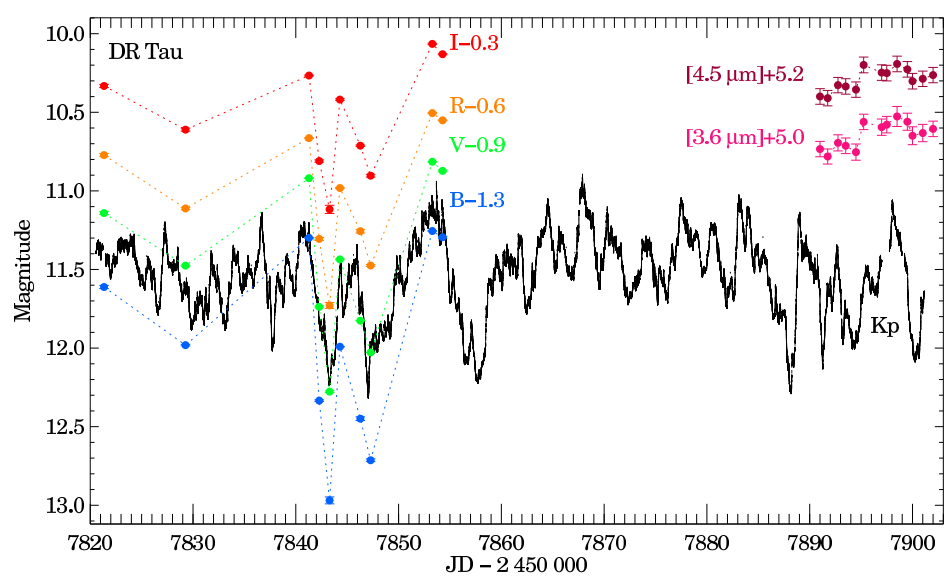

Figure 1. Multiwavelength light curves of DR Tau

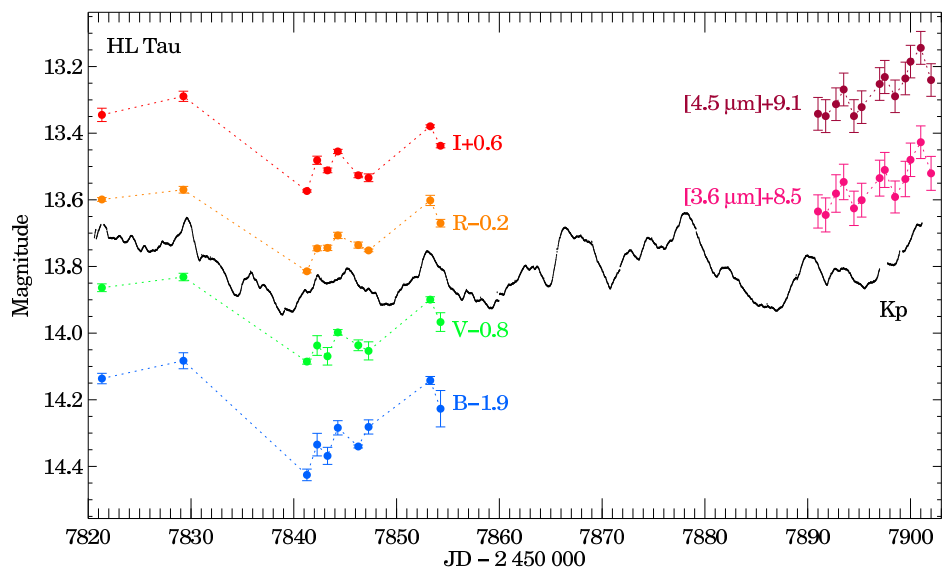

Figure 2. Multiwavelength light curves of HL Tau

Our ground-based multifilter photometry revealed that the shape of the light curves are similar at all wavelengths, although the amplitude of the variability may differ. By plotting the data points as a function of the Kp magnitude, the plots could be well fitted with a line whose slope gives the variability amplitude relative to the Kp band. By comparing the measured amplitudes to the standard interstellar extinction law (Cardelli et al. 1989), we can exclude variable extinction as the cause of the variability. A possible explanation is variable accretion. The accretion hot spot on the star causes the optical variability, while the changing illumination causes variability of the inner disk emission observable at the Spitzer wavelengths.

The variability amplitude of DR Tau decreases with increasing wavelength, from which we suggest that part of the inner disk may be optically thick and invariable, hence the smaller infrared amplitudes compared to the optical ones. The variability amplitudes of HL Tau is similar at all wavelengths. We propose that the infrared emission may be dominated by optically thin material (such as halo or envelope), which closely follows the variability of the illuminating central source.

Acknowledgements. This project has received funding from the European Research Council (ERC) under the European Union's Horizon 2020 research and innovation programme under grant agreement No 716155 (SACCRED). 


\section{References}

Herbst, W., Herbst, D. K., Grossman, E. J., \& Weinstein, D. 1994, AJ, 108, 5

Morales-Calderón, M, Stauffer, J. R., Hillenbrand, L. A. et al. 2011, ApJ, 733, 50

Sicilia-Aguilar, A., Banzatti, A., Carmona, A. et al. 2016, PASA, 33, 59

Cardelli, J. A., Clayton, G. C. \& Mathis, J. S. 1989, ApJ, 345, 245 